\title{
Selecting short length nucleic acids localized in exosomes improves plasma EGFR mutation detection in NSCLC patients
}

\author{
Yoonjung Kim ${ }^{\dagger}$, Saeam Shin ${ }^{\dagger}$, Boyeon Kim and Kyung-A Lee* (i)
}

\begin{abstract}
Background: Exosomal nucleic acid (exoNA) is a feasible target to improve the sensitivity of EGFR mutation testing in non-small cell lung cancer patients with limited cell-free DNA (cfDNA) mutant copies. However, the type and size of target exONA related to the sensitivity of EGFR mutation testing has not been explored extensively.

Methods: The type and size of target exoNA related to the sensitivity of EGFR mutation testing was evaluated using ddPCR. A total of 47 plasma samples was tested using short-length exoTNA (exosomal DNA and RNA) and cfDNA.

Results: The sensitivity of short-length exoTNA (76.5\%) was higher than that of cfDNA (64.7\%) for detecting EGFR mutations in NSCLC patients. In EGFR-mutant NSCLC patients with intrathoracic disease (MO/M1a) or cases with low-copy T790M, the positive rate was $63.6 \%(N=7 / 11)$ and $45.5 \%(N=5 / 11)$ for short-length exoTNA and cfDNA, respectively. On average, the number absolute mutant copies of short-length exoTNA were 1.5 times higher than that of cfDNA. The mutant allele copies (Ex19del and T790M) in short-length exoTNA were relatively well preserved at 4 weeks after storage. The difference (\%) in absolute mutant allele copies (Ex19del) between 0 days and 4 weeks after storage was $-61.0 \%$ for cfDNA.
\end{abstract}

Conclusion: Target nucleic acids and their size distribution may be critical considerations for selecting an extraction method and a detection assay. A short-length exoTNA (200 bp) contained more detectable tumor-derived nucleic acids than exoDNA ( 200 bp length or a full-length) or cfDNA. Therefore, a short-length exoTNA as a sensitive biomarker might be useful to detect EGFR mutants for NSCLC patients with low copy number of the mutation target.

Keywords: Liquid biopsy, Extracellular vesicles, Circulating tumor DNA, Non-small cell lung cancer, Epidermal growth factor receptor, ddPCR

\section{Background}

The identification of driver and resistance mutations located in the tyrosine kinase domain of EGFR in a subset of non-small cell lung cancer (NSCLC) is necessary to guide patient treatment options and to predict prognosis [1-3]. The majority of EGFR genotyping is assessed through conventional tumor biopsy. However, this process can frequently put the patient at risk and may miss portions of the tumor that are developing treatment

\footnotetext{
*Correspondence: KAL1119@yuhs.ac

${ }^{\dagger}$ Yoonjung Kim and Saeam Shin contributed equally to this work

Department of Laboratory Medicine, Yonsei University College

of Medicine, Seoul, Republic of Korea
}

resistance or have acquired new driver mutations due to tumor heterogeneity [4]. Thus, liquid biopsy is considered an alternative for detecting resistant-EGFR mutants in NSCLC patients undergoing tyrosine kinase inhibitor (TKI) therapy [5]. Circulating tumor DNA (ctDNA) is the most common source of nucleic acid for detecting EGFR and is already being implemented in clinical practice [6]. Current technologies, such as polymerase chain reaction (PCR)-based method and next-generation sequencing, have been developed to detect very low level of mutant ctDNA fraction in plasma [7, 8]. However, these platforms show varying sensitivity depending on mutations and are not satisfactory enough to get reliable results in patients with scant T790M copies $(<0.01 \%)$ [9-11]. 
Since tumor-derived nucleic acids rarely exist existing in patient's plasma, pre-analytical steps, especially selecting target materials that harbored relatively abundant mutant alleles and extracted them using an appropriated method, are important to improve the sensitivity of EGFR test using patient's plasma with low levels of mutant alleles.

Exosomes are endosome-derived small membranebound vesicles that are released by different cell types [12]. Exosomes carry proteins, lipids, and nucleic acids, including DNAs and RNAs [13]. Growing evidence have indicated that exosomes are associated with tumor development and metastasis [13, 14]. Exosomal nucleic acids (exoNA) containing tumor-derived nucleic acids were studied as a target for cancer mutation testing, and they also emerged as biomarkers for cancer diagnosis [15]. Moreover, they have recently been reported to be a sensitive source for $E G F R$ genotyping [16, 17]. To improve the sensitivity of detection in patients with limited mutant copies of cfDNA, such as those with early-stage NSCLC or intrathoracic disease (M0/M1a), exosomal nucleic acid (exoNA) might be a feasible alternative $[15,18]$. To acquire high quality and quantity of exoNAs, the type and size of target exoNA should be considered when choosing an extraction method; however, the type and size of target exoNA related to the sensitivity of EGFR mutation testing have not been extensively studied. In previous studies, the target of exoNA was exoDNA, exoRNA, or combined exoDNA/RNA [15-17, 19, 20]. Some studies targeted high molecular DNA from exosomes [17, 21], and other studies focused on fragmented DNA that localized in exosomes [22]. In this study, we evaluated exoNAs to carefully determine a sensitive circulating biomarker in a plasma EGFR genotyping assay. Our results demonstrate that short-length exoTNA (exosomal DNA and RNA) is a feasible target in patients with low-level EGFR mutant copies. We extracted short-length exoTNA using specific extraction kits that could enrich tumor-derived $\sim 200$-bp-sized NAs. We then compared the ExoNAs and cfDNA isolated from NSCLC patients using droplet digital PCR (ddPCR) to detect EGFR mutations including exon 19 deletion (Ex19del), T790M, and L858R.

\section{Materials and methods Study design}

ddPCR assays were performed with the PrimePCR ${ }^{\mathrm{TM}}$ $\mathrm{ddPCR}^{\mathrm{TM}}$ Mutation Detection Assay kit and Prime$\mathrm{PCR}^{\mathrm{TM}} \mathrm{ddPCR}^{\mathrm{TM}}$ EGFR Exon 19 Deletions Screening Kit (Bio-Rad Laboratories, Hercules, CA, USA) (Additional file 1: Table S1). We selected three hotspot mutations of Ex19del, L858R, and T790M. L858R and Ex19del are the most common forms of EGFR sensitizing mutations (85\%) that are responsive for EGFR TKI treatment [23]. In case of progression on 1st generation TKI treatment,
T790M mutation testing is recommended as acquired T790M mutation is the most common resistance mechanism $(>50 \%)$ that is responsive for 3rd generation TKI treatment $[23,24]$. The limitation of detection (LOD) was determined as the lowest mutant concentration above the $95 \%$ confidence interval (CI) of the wild-type (WT) control, which was determined using a Poisson model (Additional file 1: Table S2). Validation ddPCR was performed using Multiplex I cfDNA Reference Standard (Horizon Discovery, Cambridge, UK) (Additional file 1: Table S3). The ability to detect EGFR mutation based on type of input nucleic acid (short-length exoTNA and a size range of exoDNA) was evaluated (Additional file 1: Figures S1 and S2). Analytic performance of isolated cfDNA and short-length exoTNA was evaluated using ddPCR. We assessed the influence on cfDNA levels and short-length exoTNA according to storage period.

\section{Patients}

From November 2017 to November 2018, 47 NCSLC patients were consented and enrolled. EGFR genotyping results $(N=46)$ from the corresponding tissue specimens were obtained. Patients consented to the protocol approved by the Institutional Review Board of Gangnam Severance Hospital and Kangnam Sacred Heart Hospital. Plasma $(2 \mathrm{~mL})$ was collected from a total of 47 patients and stored at $-80^{\circ} \mathrm{C}$.

\section{Nucleic acid extraction and CDNA synthesis}

We extracted plasma cfDNA using the MagMAX CellFree DNA Isolation Kit (Thermo Fisher Scientific, Waltham, MA, USA). Exosomes were isolated from plasma using ExoQuick ${ }^{\mathrm{TM}}$ (System Biosciences, Mountain View, CA, USA). Subsequently, short-length exoDNA and short-length exoTNA were isolated by MagMAX Cell-Free DNA Isolation Kit (Thermo Fisher Scientific) and MagMAX ${ }^{\mathrm{TM}}$ Total Nucleic Acid Isolation Kit (Thermo Fisher Scientific), respectively. A size range of exoDNA was isolated by the QIAamp DNA Blood Mini Kit (Qiagen, Hilden, Germany). The concentration and size distribution of cfDNA and exoNA were assessed using a 2200 TapeStation Instrument (Agilent Technologies, Santa Clara, CA, USA) with the Agilent High Sensitivity D1000 ScreenTape System and Genomic DNA ScreenTape System. The RNA yield and size distribution were analyzed using an Agilent 2100 Bioanalyzer with an RNA 6000 Pico kit (Agilent Technologies, Foster City, CA, USA). cDNA synthesis was performed using a SuperScript ${ }^{\mathrm{TM}}$ VILO $^{\mathrm{TM}}$ cDNA Synthesis Kit (Invitrogen, Carlsbad, CA, USA). 


\section{Validation of ddPCR}

The ddPCR assays were performed with the Prime$\mathrm{PCR}^{\mathrm{TM}} \mathrm{ddPCR}^{\mathrm{TM}}$ Mutation Detection Assay kit and PrimePCR ${ }^{\mathrm{TM}} \mathrm{ddPCR}^{\mathrm{TM}}$ EGFR Exon 19 Deletions Screening Kit (Bio-Rad Laboratories) (Additional file 1: Table S1). We used cfDNA from Multiplex I cfDNA Reference Standards (Horizon Discovery) that included wild-type cfDNA with mutant allele frequencies of $5 \%, 1 \%$, and $0.1 \%$. cfDNA Reference Standards (Horizon Discovery) with $0.1 \%$ mutant allele was serially diluted to wild-type cfDNA for analytical sensitivity of the ddPCR assay (Additional file 1: Table S3). Healthy control samples and DNA-free samples were also analyzed (Additional file 1: Table S2) [25, 26]. Amplifications were carried out in a reaction volume of $20 \mu \mathrm{L}$ on a QX100 Droplet Digital PCR System (Bio-Rad). The $20 \mu \mathrm{L}$ PCR mix was composed of $10 \mu \mathrm{L}$ Bio-Rad Super mix TaqMan, 1-2 $\mu \mathrm{L}$ of each amplification primer/ probe mix, and 8-9 $\mu \mathrm{L}$ NAs. Thermal cycling comprised an initial denaturing and polymerase hot-start activating step of $10 \mathrm{~min}$ at $95^{\circ} \mathrm{C}$, followed by 40 cycles of $95{ }^{\circ} \mathrm{C}$ for $30 \mathrm{~s}$ and $55^{\circ} \mathrm{C}$ for $60 \mathrm{~s}$. Results were analyzed with QuantaSoft v.1.7.2 software (Bio-Rad) and reported as copies per milliliter of plasma.

\section{Effects of storage on cfDNA and short-length exoTNA concentrations}

Samples from two patients with EGFR mutation and three normal controls were collected in $K_{2}$ EDTA tubes. Immediately separated plasma was aliquoted into three tubes per sample and stored at $-80^{\circ} \mathrm{C}$. We assessed the influence of storage period on cfDNA levels and shortlength exoTNA extracted at different time points $(0,14$, and 28 days).

\section{Data analysis}

Quantification of the number of target DNA molecules in the reaction is achieved by counting the number of positive and negative droplets. The LOD was determined as the lowest mutant concentration above the $95 \%$ CI of the WT control. The $95 \%$ CI was determined using a Poisson model and CLSI EP17-A2 [26, 27]. Details are described in Additional file 1: Table S2. Assays were considered "positive" if the measured event rate was $\geq 2$ events/assay and "negative" if the event rate within a gated region was $<2$ events/assay.

\section{Statistical analyses}

Statistical analysis was performed using R (version 3.5.2, http://www.r-project.org) and MedCalc software (https ://www.medcalc.org/). Data are presented using a 95\% CI and 2 -sided $P$ value.

\section{Results \\ Patient characteristics}

The patients' characteristics are described in Table 1. Patients had a median age of 73 years (range, $52-85$ years), and 19 patients $(40.4 \%)$ were female. The adenocarcinoma, squamous cell carcinoma, and other histologic types numbered 32 (68.1\%), 9 (19.1\%), and 6 (12.8\%), respectively. Stage IV was dominant $(N=31$, $66.0 \%$ ), and other stages (I-III) represented $34.0 \%$ of total patients. Patients with intrathoracic metastatic disease (M0/M1a) accounted for $51.0 \%$ (Table 1).

\section{Assessment of ddPCR assay sensitivity}

The analytical sensitivity of the ddPCR assay was evaluated using spiked samples with mutant allele frequencies of $1 \%, 0.1 \%, 0.02 \%, 0.01 \%$, and $0.005 \%$ (Additional file 1: Table S3). The expected copy number of mutant alleles (1-32 copies) spiked into the wild-type alleles

Table 1 Baseline characteristics of patients

\begin{tabular}{|c|c|}
\hline Characteristic & All patients $(N=47)^{\mathrm{a}}$ \\
\hline Age (years) & $73(52-85)$ \\
\hline \multicolumn{2}{|l|}{ Gender } \\
\hline Female & $19(40.4 \%)$ \\
\hline Male & $28(59.6 \%)$ \\
\hline \multicolumn{2}{|l|}{ Histologic type } \\
\hline Adenocarcinoma & $32(68.1 \%)$ \\
\hline Squamous cell carcinoma & $9(19.1 \%)$ \\
\hline Other & $6(12.8 \%)$ \\
\hline \multicolumn{2}{|l|}{ Tumor stage } \\
\hline 1 & $5(10.6 \%)$ \\
\hline$\|$ & $2(4.3 \%)$ \\
\hline III & $9(19.1 \%)$ \\
\hline IV & $31(66.0 \%)$ \\
\hline \multicolumn{2}{|l|}{ M category ${ }^{b}$} \\
\hline Mo & $16(34.0 \%)$ \\
\hline M1a & $8(17.0 \%)$ \\
\hline M1b & $3(6.4 \%)$ \\
\hline M1c, single organ & $3(6.4 \%)$ \\
\hline M1c, multi organs & $17(36.2 \%)$ \\
\hline \multicolumn{2}{|l|}{ Chemotherapy } \\
\hline TKI-naïve & $43(91.5 \%)$ \\
\hline TKI-treated & $4(8.5 \%)$ \\
\hline \multicolumn{2}{|c|}{ Other not otherwise specified, $T K I$ tyrosine kinase inhibitor } \\
\hline \multicolumn{2}{|c|}{ a Results are expressed as median (range) or number (\%) } \\
\hline \multicolumn{2}{|c|}{$\begin{array}{l}\text { b According to the 8th TMN edition, M1a indicates lung metastases or pleural/ } \\
\text { pericardial malignant effusion or nodules; M1b indicates a single metastatic } \\
\text { lesion in a single distant organ; M1c indicates multiple lesions in a single organ } \\
\text { or multiple lesions in multiple organs }\end{array}$} \\
\hline
\end{tabular}


(2000-20,000) and the actual copy number of mutant alleles observed in the spiked samples are shown in Additional file 1: Table S3. An ultra-rare mutation (1 copy in a spiked sample) as low as $0.007-0.008 \%$ was successfully detected by the ddPCR assay. However, when we considered 2 copies $/ \mathrm{mL}$ as a threshold for a positive result, Ex19del, L858R, and T790M were detected even at fractional abundance of $0.03 \%, 0.013 \%$, and $0.018 \%$, respectively (Additional file 1: Table S3). The limit of blank (LOB) defined by the frequency of positive droplets measured in DNA-free samples and the standard deviation (SD) of healthy controls were used to determine the lower LOD. Additional file 1: Table S2 shows the raw data for LOB analysis and LOD.

\section{Comparison between cfDNA and size-selectively extracted exoNAs}

To elucidate the components of exoNAs that were related to the sensitivity of EGFR mutation testing, pooled plasma samples with Ex19del mutation were used. Isolated cfDNA, short-length exoNAs (DNA and TNA), and a full-length exoDNA including low and high molecular weight nucleic acids were co-isolated (Additional file 1: Figure S1). We assessed tumor-derived NAs to be more abundant in the shortlength NAs ( 200 bp long) than the full-length exoDNAs in exosomes (Additional file 1: Figures S1 and S2). Especially, short-length exoTNA is superior to other nucleic acid materials (cfDNA, short-length exoDNA, and full-length exoDNA) for detecting EGFR mutant alleles (Additional file 1: Figure S1). Quantity and size of short-length NAs were further confirmed by Bioanalyzer results, which also showed a major peak at less than $\sim 200$ bp long (data not shown).

\section{Mutant allele ratio between cfDNA and short-length exoNA}

We evaluated the quantity of wild-type and mutant allele copies at cfDNA and short-length exoNAs (DNA and TNA) in spiked samples from NSCLC patients harboring EGFR mutation. The mutant allele ratio (shortlength exoTNA/cfDNA) ranged from 1.2 to 2.5 , and the mutant allele ratio of short-length exoDNA/cfDNA ranged from 0.0 to 1.0 (Table 2). Short-length exoTNA showed the largest absolute number of mutant allele copies compare to cfDNA and short-length exoDNA. However, due to the abundance of wild-type allele copies in short-length exoTNA, the mutant allele fraction (\%) of cfDNA was generally higher than that of shortlength exoTNA. Both cfDNA and short-length exoTNA are considered good materials for detection of tumorderived mutant alleles (Table 2).

We performed ddPCR using 250, 500, 750, and $1000 \mu \mathrm{L}$ plasma to assess the proper plasma volume for sensitive detection and monitoring. The amount of input plasma volume determined number of mutant allele copies in cfDNA and exoNAs. The mutant allele ratio between cfDNA and short-length exoTNA was relatively higher in a small input volume $(250 \mu \mathrm{L})$ (Additional file 1: Figure S1).

Table 2 Comparison between cfDNA and short-length exosomal nucleic acids

\begin{tabular}{|c|c|c|c|c|c|c|c|c|}
\hline \multirow[t]{2}{*}{ Sample $^{a}$} & \multirow[t]{2}{*}{ Target } & \multirow{2}{*}{$\begin{array}{l}\text { Mutant } \\
\text { events }\end{array}$} & \multirow{2}{*}{$\begin{array}{l}\text { Wild-type } \\
\text { events }\end{array}$} & \multirow[t]{2}{*}{ Fraction (\%) } & \multicolumn{2}{|c|}{ Mutant allele ratio } & \multicolumn{2}{|c|}{ Wild-type allele ratio } \\
\hline & & & & & $\begin{array}{l}\text { exoDNA/ } \\
\text { cfDNA }\end{array}$ & $\begin{array}{l}\text { exoTNA/ } \\
\text { cfDNA }\end{array}$ & $\begin{array}{l}\text { exoDNA/ } \\
\text { cfDNA }\end{array}$ & $\begin{array}{l}\text { exoTNA/ } \\
\text { cfDNA }\end{array}$ \\
\hline \multirow[t]{3}{*}{ Spiked sample 1 (Ex19del) } & cfDNA & 20 & 418 & 4.78 & 0.5 & 1.2 & 0.6 & 2.7 \\
\hline & Short-length-exoDNA & 9 & 270 & 3.33 & & & & \\
\hline & Short-length-exoTNA & 24 & 1138 & 2.11 & & & & \\
\hline \multirow[t]{3}{*}{ Spiked sample 2 (L858R) } & cfDNA & 1 & 156 & 0.64 & 1 & 2 & 0.8 & 2.6 \\
\hline & Short-length-exoDNA & 1 & 131 & 0.76 & & & & \\
\hline & Short-length-exoTNA & 2 & 399 & 0.5 & & & & \\
\hline \multirow[t]{3}{*}{ Spiked sample 3 (L858R) } & cfDNA & 2 & 38 & 5.26 & 0 & 2.5 & 0 & 6 \\
\hline & Short-length-exoDNA & 0 & 1 & 0 & & & & \\
\hline & Short-length-exoTNA & 5 & 228 & 2.19 & & & & \\
\hline \multirow[t]{3}{*}{ Spiked sample 3 (T790M) } & cfDNA & 0 & 97 & 0 & N.A. & N.A. & N.A. & N.A. \\
\hline & Short-length-exoDNA & 6 & 89 & 6.74 & & & & \\
\hline & Short-length-exoTNA & 8 & 307 & 2.61 & & & & \\
\hline
\end{tabular}

cfDNA cell-free DNA, exoDNA exosomal DNA, exoTNA exosomal DNA and RNA, Ex19del exon 19 deletion, N.A not available

a Spiked samples with pooled plasma from patients harboring mutations in EGFR (Ex19del, L858R, and T790M). cfDNA and short-length exoDNA were extracted using MagMAX Cell-Free DNA Isolation Kit. Short-length exoTNA was extracted using MagMAX ${ }^{\mathrm{TM}}$ Total Nucleic Acid Isolation Kit 


\section{Quantification of short-length exoTNA and cfDNA in clinical samples}

Short-length exoNA and cfDNA were extracted from equal volumes $(1 \mathrm{~mL})$ of plasma samples from 47 patients. Among them, 7 samples were not available for NA quantification due to lack of volume. The concentrations of short-length exoTNA and cfDNA are depicted in Fig. 1. The median concentration levels of short-length exoTNA and cfDNA were $11.9 \mathrm{ng} / \mathrm{mL}$ and $6.1 \mathrm{ng} / \mathrm{mL}$, respectively, and there was not a significant difference between short-length exoTNA and cfDNA $(P=0.6978)$.

\section{Comparison of EGFR mutation status in NSCLC patients}

Plasma samples from 47 patients were tested with shortlength exoTNA and cfDNA using ddPCR, and each sample was analyzed for Ex19del, L858R, and T790M. Of the

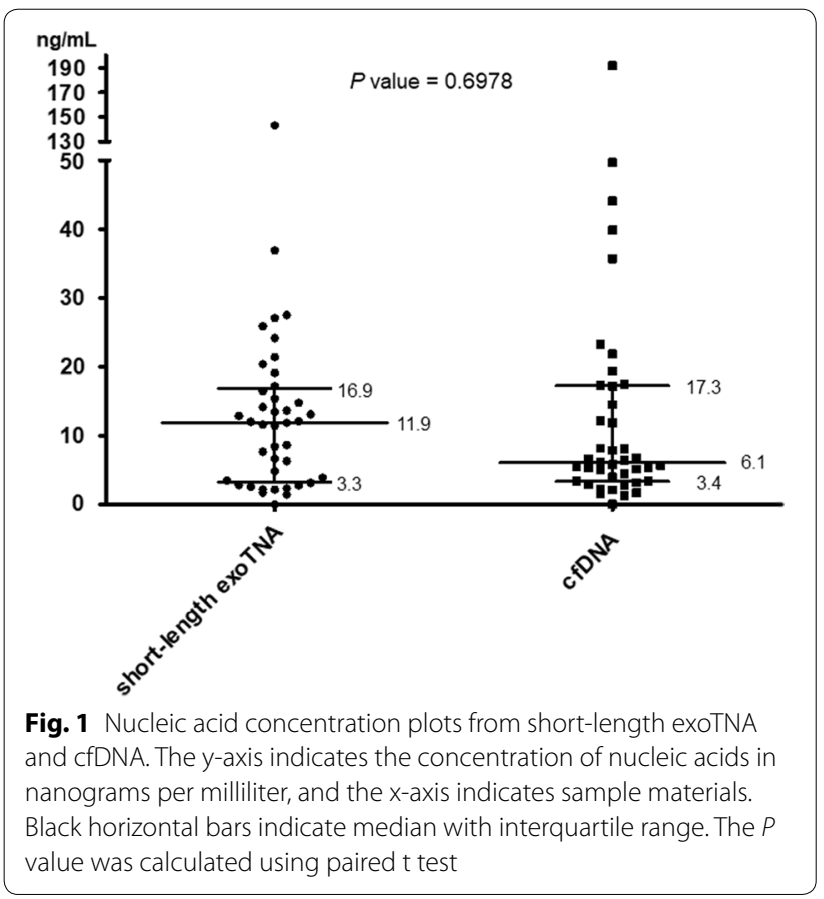

141 measurements ( 3 mutant sites per each patient), 17 and 124 measures were classified as true positives (TP) and true negatives (TN), respectively, compared to tissue biopsy (Table 3). Seventeen TPs were detected from 15 patients; among them, 2 patients harbored both activating and resistant EGFR mutations (Fig. 2b). Three patients harboring T790M or L858R were only detected in plasma samples using the cobas ${ }^{\circledR} E G F R$ Mutation Test v2 not in tissue samples. These EGFR mutations were also detected in cfDNA or/and short-length exoTNA up to a 9.5\% mutant allele fraction (\%). Therefore, we regarded these three mutations as "TP" results. The sensitivity of short-length exoTNA (76.5\%) was higher than that of cfDNA (64.7\%) for activating/resistant EGFR mutations (Table 3). We also found that $46.7 \%$ (7/15) of TP cases were from patients with intrathoracic (M0/M1a) disease, and $53.3 \%(8 / 15)$ were from patients with M1b/M1c disease. In false negative (FN) results, $9 \mathrm{FNs}$ (short-length exoTNA, 4 samples; cfDNA, 5 samples) were from five patients with M0/M1a. One FN case (cfDNA, 1 sample) was from a patient who progressed under treatment with an EGFR-TKI (Fig. 2b). The specificity of EGFR genotyping was $100.0 \%$ and $97.6 \%$ for short-length exoTNA and cfDNA, respectively (Table 3). Two false-positives (FPs) were found only in cfDNA with two events of the mutant allele in ddPCR. The accuracy of EGFR genotyping was slightly higher with short-length exoTNA (97.2\%) than with cfDNA (93.6\%) (Table 3). The mean mutant allele ratio (short-length exoTNA / cfDNA) was 1.5 and ranged from 0.8 to 6.6 (Fig. 2a, b). The main peak of nucleic acids of cfDNA and short-length exoTNA was $\sim 200 \mathrm{bp}$ long in EGFR mutated NSCLC patient plasma (Fig. 2c).

\section{Effects of storage at cfDNA and short-length exoTNA}

We determined whether the storage duration of cfDNA and short-length exoTNA could affect the detection of EGFR mutations. The median wild-type allele ratio (short-length exoTNA/cfDNA) of three normal controls was $0.9,1.2$ and 0.7 at 0 days, 2 weeks, and 4 weeks,

Table 3 Comparison of the EGFR mutation status between tumor tissue and plasma in NSCLC patients $(N=47)$

\begin{tabular}{|c|c|c|c|c|c|}
\hline \multirow[t]{2}{*}{ EGFR genotype } & \multirow[t]{2}{*}{ TP and $T^{a}$} & \multicolumn{2}{|l|}{ cfDNA } & \multicolumn{2}{|l|}{ Short-length exoTNA } \\
\hline & & Mutant type & Wild-type & Mutant type & Wild-type \\
\hline Mutant type & 17 & 11 & 6 & 13 & 4 \\
\hline Wild-type & 124 & 3 & 121 & 0 & 124 \\
\hline Sensitivity,\% (95\% Cl) & & $64.7 \%(38.3-85.8 \%)$ & & $76.5 \%(50.1-93.2 \%)$ & \\
\hline Specificity, \% (95\% Cl) & & $97.6 \%(93.1-99.5 \%)$ & & $100.0 \%$ (97.1-100.0\%) & \\
\hline Accuracy, \% (95\% Cl) & & $93.6 \%(88.2-97.0 \%)$ & & $97.2 \%(92.9-99.2 \%)$ & \\
\hline
\end{tabular}

NSCLC non-small cell lung cancer, $T P$ true positive, $T N$ true negative, $C l$ confidence interval

a Tissue EGFR genotyping results were considered 'true positive' or 'true negative.'Three cases for which tissue EGFR was negative showed positive results using the cobas $^{\circledR}$ EGFR Mutation Test v2. These results were regarded as 'true positive.' 


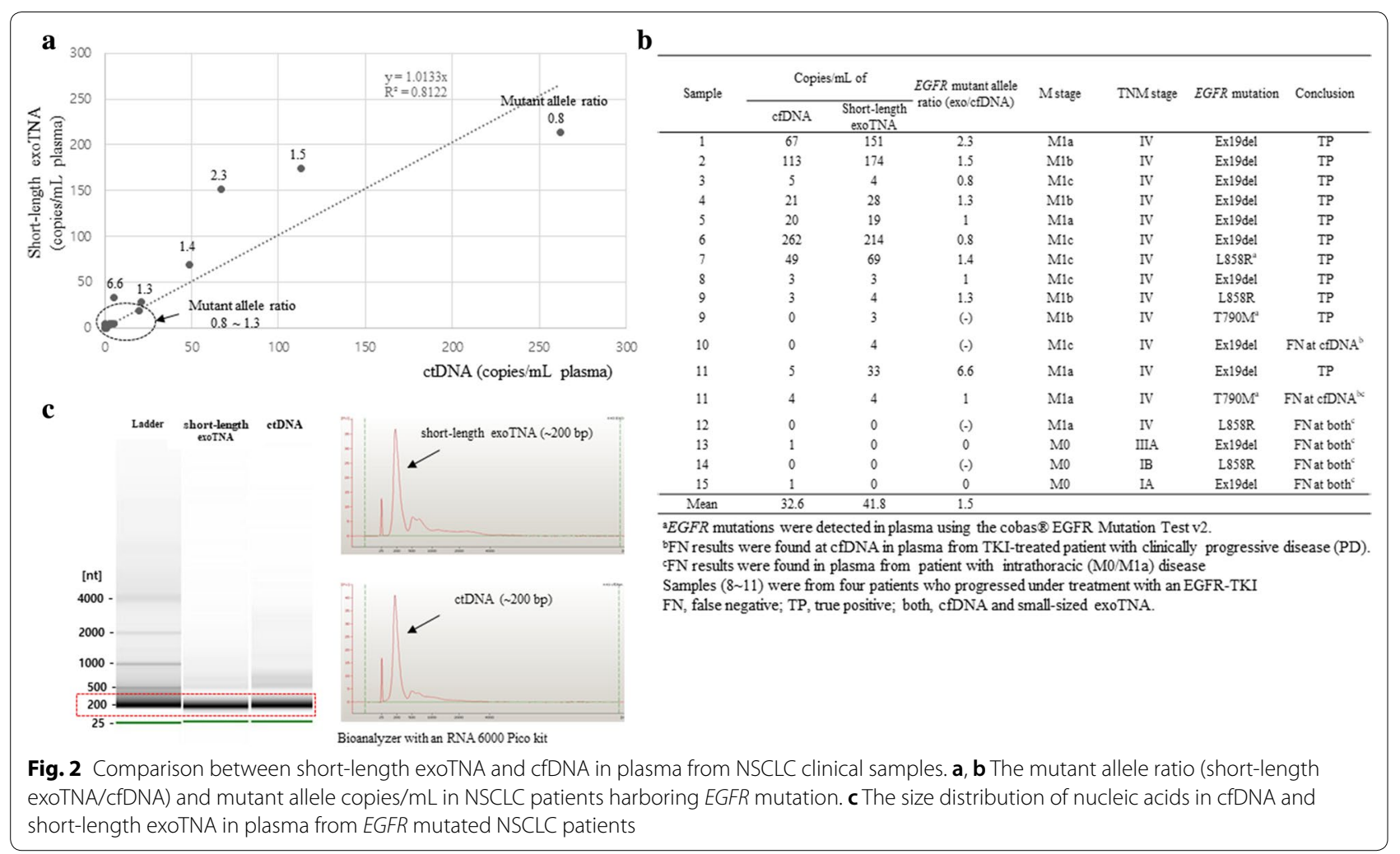

respectively. The average wild-type allele ratio in two patients was $1.8,3.3$, and 2.3 at 0 days, 2 weeks, and 4 weeks, respectively. The average mutant allele ratio was 1.6, 6.6, and 2.8 at 0 days, 2 weeks, and 4 weeks, respectively. The T790M mutant allele ratio showed a fivefold increase after 2 weeks of storage, and this mutant allele ratio at 4 weeks later was similar to that of day 0 (Fig. 3a). The mutant allele copies (Ex19del and T790M) in shortlength exoTNA were relatively well preserved after 4 weeks (Fig. 3b). The difference (\%) between Ex19del mutant allele fraction (\%) and that of the absolute mutant allele copies between 0 days and 4 weeks after storage was $-21.3 \%$ and $-61.0 \%$, respectively, in cfDNA (Fig. $3 b$ ).

\section{Discussion}

To improve the sensitivity of low levels of plasma cfDNA, there has been a focus on enriching for cfDNA fragments on the basis of length [28-30]. It has been reported that mutant alleles occur more commonly in shorter fragments of cfDNA in lung cancer patients [30]. However, the size of NA in exosomes that might contain relatively high tumor-derived NAs remains unclear. According to Hur et al., EGFR-mutant DNA is mainly distributed as high-molecular weight DNA $(\sim 10 \mathrm{~kb})$ in exosomes from plasma and bronchoalveolar lavage fluid [17]. However, they only evaluated NAs of exosomes using an extraction kit for full-length DNA. Fernando et al. demonstrated that fragmented DNAs of $\sim 200 \mathrm{bp}$ in size comprised the majority of NAs localized in exosomes [22]. By comparing different sizes of exoNAs, we observed that the EGFR mutant alleles were more enriched in short-length NAs ( 200 bp long) than in high-molecular weight DNA in exosomes (Additional file 1: Figures S1 and S2).

Recent studies have shown that using single-step isolation of combined exosomal RNA/DNA and cfDNA is a highly sensitive method for detecting EGFR mutations in NSCLC patient plasma $[16,18]$. However, the target NAs and their size have not been clearly elucidated. We determined that short-length exoTNA is superior to other nucleic acid materials (cfDNA, short-length exoDNA and full-length exoDNA) for mutant allele detection (Table 2 and Fig. 2). When comparing short-length exoTNA to cfDNA, the average number of mutant copies is 1.5 times higher for short-length exoTNA (Fig. 2a). Furthermore, the sensitivity of detecting EGFR mutations using shortlength exoTNA is higher than that using cfDNA $(76.5 \%$ vs. $64.7 \%$ ) (Table 3 ).

Analysis of cfDNA from blood could be an alternative method for identifying EGFR mutations in NSCLC patients. However, detectable mutant copies in cfDNA are too few to increase false negative rate in EGFRmutated NSCLC. Patients with low T790M copy number 

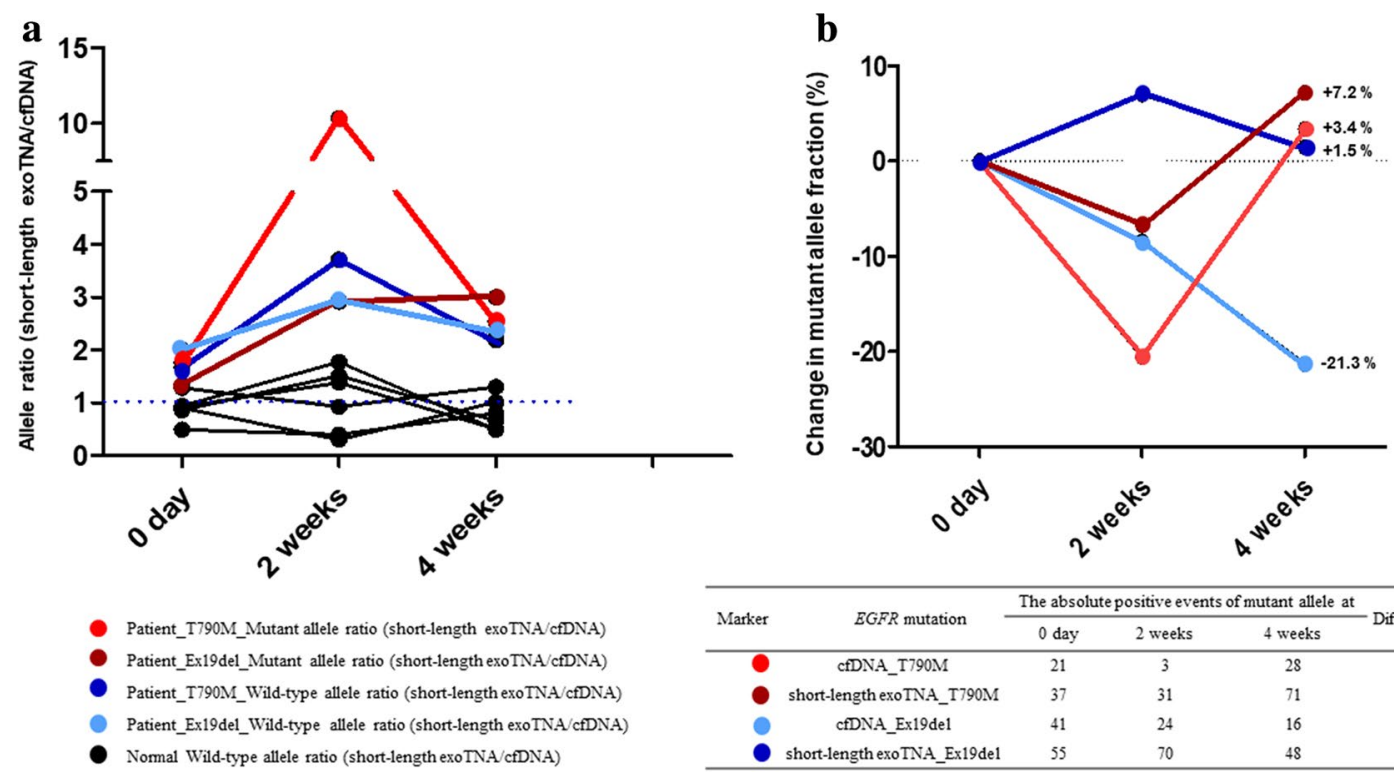

\begin{tabular}{|c|c|c|c|c|c|}
\hline \multirow{2}{*}{ Marker } & \multirow{2}{*}{ EGFR mutation } & \multicolumn{3}{|c|}{ The absolute positive events of mutant allele at } & \multirow{2}{*}{ Difference $(\%)^{2}$} \\
\hline & & 0 day & 2 weeks & 4 weeks & \\
\hline O & cfDNA_T790M & 21 & 3 & 28 & 33.3 \\
\hline - & short-length exoTNA_T790M & 37 & 31 & 71 & 91.9 \\
\hline - & cfDNA_Ex19de1 & 41 & 24 & 16 & -61.0 \\
\hline - & short-length exoTNA_Ex $19 \mathrm{de} 1$ & 55 & 70 & 48 & -12.7 \\
\hline
\end{tabular}

Fig. 3 Effects of storage on cfDNA and short-length exoTNA. a Allele ratio (short-length exo/cfDNA) change in two patients with EGFR mutation and three normal controls. In normal controls, only the wild-type allele ratio (short-length exo/cfDNA) is depicted. b The change in mutant allele fraction (\%) in short-length exoTNA and cfDNA according to storage time

$(<10$ copies $/ \mathrm{mL})$ have a similar response to osimertinib to patients with a higher T790M copy number $(\geq 10$ copies/mL) [31]. Furthermore, an actual number of patients with low T790M copy number $(<10$ copies/mL) was fourfold higher than the number of patients with a higher T790M copy number ( $\geq 10$ copies/mL) [32]. Around half of cases in the present study harboring plasma EGFR mutation also had low mutant allele copies $(<10$ copies/mL) (Fig. 2a, b). In EGFR-mutant NSCLC patients with intrathoracic disease (M0/M1a) or cases with low copy $\mathrm{T} 790 \mathrm{M}$, the positive rate was $63.6 \%(N=7 / 11)$ and $45.5 \%(N=5 / 11)$ in short-length exoTNA and cfDNA, respectively (Fig. 2b). These data demonstrate that the increased numbers of detectable EGFR mutant copies obtained from extraction of exoTNA could influence the sensitivity of ddPCR-based EGFR mutation test for cases with low copy EGFR mutants.

Short-length exoTNA and cfDNA showed generally good concordance with tissue EGFR results. Despite the small number of studied samples, $17.6 \%$ of $E G F R$ mutants $(N=3 / 17)$ were detected in plasma samples where the mutation was not detected in tissue previously. The false-negative results of tissue might be caused by tumor heterogeneity [33] or failure to obtain adequate specimen. This highlights the feasibility of blood-based EGFR testing in diagnosis and monitoring of cancer.

To evaluate the influence of storage period on cfDNA and short-length exoTNA for assay performance, we compared cfDNA and exoTNA from plasma at different time points $(0,14$, and 28 days $)$. When plasma was stored at $-80{ }^{\circ} \mathrm{C}$ for 4 weeks, the amounts of both T790M and Ex19del mutant copies in short-length exoTNA remained stable; however, in cfDNA, Ex19del mutant copies decreased by up to $61 \%$. In the previous study, storage at $-20{ }^{\circ} \mathrm{C}$ barely impacted the overall amounts of exosomal miRNAs for at least 5 years [34]. The stability of exoTNA could be explained by the mechanism by which lipid bilayer membrane coating protects internal DNA and RNA [21]. The amounts and integrity of cfDNA could be affected by storage duration. Storing plasma samples at $-80{ }^{\circ} \mathrm{C}$ is recommended until further processing of cfDNA isolation is implemented [35]. Following the recommendation, a majority of laboratories have stored plasma at $-80{ }^{\circ} \mathrm{C}$ [36]. Barrett et al. demonstrated the stability of cfDNA from plasma samples stored at $-80{ }^{\circ} \mathrm{C}$ for up to 2 weeks [35]. However, according to our data, the storage period can influence the stability of cfDNA that is stored more than 2 weeks. Therefore, when performing the EGFR assay, the storage duration of plasma should be considered. Given the limited sample size we used, future study should be performed to confirm the effects of $-80{ }^{\circ} \mathrm{C}$ storage on the amount and integrity of cfDNA and short-length exoTNA. 


\section{Conclusion}

Target nucleic acids and their size distribution might be critical considerations for selecting an extraction method and a detection assay. In this study, we mainly determined the characteristics of an effective target component in exosomes. A shorter exoTNA with 200 bp length contained more detectable tumorderived nucleic acids than exoDNA ( $~ 200$ bp length or full-length) or cfDNA. Blood-based cancer diagnostic testing is a promising tool not only for early diagnosis of cancer, but also for patient stratification and longitudinal monitoring of residual tumors [5, 37, 38]. Therefore, short-length exoTNA as a sensitive biomarker might be useful to detect EGFR mutants for NSCLC patients with low copy numbers of the mutation target.

\section{Supplementary information}

Supplementary information accompanies this paper at https://doi. org/10.1186/s12935-019-0978-8.

Additional file 1: Table S1. Characteristics of the primers and probes as provided by the manufacturer. Table S2. The LOD of the ddPCR assay. Table S3. Analytical sensitivity of the ddPCR assay. Figure S1. Assessment of size-selective target exoNAs related to the sensitivity of EGFR mutation testing. Figure $\mathbf{S 2}$. The distribution of isolated nucleic acids.

\section{Abbreviations}

NSCLC: non-small cell lung cancer; TKI: tyrosine kinase inhibitor; ctDNA: circulating tumor DNA; exoNA: exosomal nucleic acid; cfDNA: cell-free DNA; exoNA: exosomal nucleic acid; exoTNA: exosomal DNA and RNA; ddPCR: droplet digital polymerase chain reaction; Ex19del: exon 19 deletion; LOD: limitation of detection; $\mathrm{Cl}$ : confidence interval; WT: wild-type; TP: true positives; TN: true negatives.

\section{Acknowledgements}

Not applicable.

\section{Author's contributions}

Designed and supervised the research, interpreted data, and reviewed and edited the manuscript: KAL; performed experiments and analysis of all samples, interpreted experimental data and wrote manuscript: YK and SS; helped to analysis of samples and evaluate the manuscript: BK. All authors read and approved the final manuscript.

\section{Funding}

Not applicable.

\section{Availability of supporting data}

The datasets supporting the conclusions of this article are included within the article and its additional files.

\section{Ethics approval and consent to participate}

All human samples are collected under Institutional Review Committee (IRB).

\section{Consent for publication}

Not applicable.

\section{Competing interests}

The authors declare that they have no competing interests.

Received: 2 June 2019 Accepted: 24 September 2019

Published online: 01 October 2019

\section{References}

1. Lynch TJ, Bell DW, Sordella R, Gurubhagavatula S, Okimoto RA, Brannigan $\mathrm{BW}$, et al. Activating mutations in the epidermal growth factor receptor underlying responsiveness of non-small-cell lung cancer to gefitinib. N Engl J Med. 2004;350:2129-39.

2. Mok TS, Wu YL, Ahn MJ, Garassino MC, Kim HR, Ramalingam SS, et al. Osimertinib or platinum-pemetrexed in EGFR T790M-positive lung cancer. N Engl J Med. 2017;376:629-40.

3. Wu YL, Zhou C, Hu CP, Feng J, Lu S, Huang Y, et al. Afatinib versus cisplatin plus gemcitabine for first-line treatment of Asian patients with advanced non-small-cell lung cancer harbouring EGFR mutations (LUX-Lung 6): an open-label, randomised phase 3 trial. Lancet Oncol. 2014;15:213-22.

4. Murtaza M, Dawson SJ, Tsui DW, Gale D, Forshew T, Piskorz AM, et al. Noninvasive analysis of acquired resistance to cancer therapy by sequencing of plasma DNA. Nature. 2013;497:108-12.

5. Molina-Vila MA. Liquid biopsy in lung cancer: present and future. Transl Lung Cancer Res. 2016;5:452-4.

6. Calabuig-Farinas S, Jantus-Lewintre E, Herreros-Pomares A, Camps C. Circulating tumor cells versus circulating tumor DNA in lung cancer-which one will win? Transl Lung Cancer Res. 2016:5:466-82.

7. Newman AM, Bratman SV, To J, Wynne JF, Eclov NC, Modlin LA, et al. An ultrasensitive method for quantitating circulating tumor DNA with broad patient coverage. Nat Med. 2014;20:548-54.

8. Diaz LA Jr, Bardelli A. Liquid biopsies: genotyping circulating tumor DNA. J Clin Oncol. 2014:32:579-86.

9. Kim Y, Shin S, Lee KA. A comparative study for detection of EGFR mutations in plasma cell-free DNA in Korean clinical diagnostic laboratories. Biomed Res Int. 2018;2018:7392419.

10. Elazezy M, Joosse SA. Techniques of using circulating tumor DNA as a liquid biopsy component in cancer management. Comput Struct Biotechnol J. 2018;16:370-8.

11. Liang Z, Cheng Y, Chen Y, Hu Y, Liu WP, Lu Y, et al. EGFR T790M ctDNA testing platforms and their role as companion diagnostics: correlation with clinical outcomes to EGFR-TKIs. Cancer Lett. 2017;403:186-94.

12. Denzer K, Kleijmeer MJ, Heijnen HF, Stoorvogel W, Geuze HJ. Exosome: from internal vesicle of the multivesicular body to intercellular signaling device. J Cell Sci. 2000;113(Pt 19):3365-74.

13. Ruivo CF, Adem B, Silva M, Melo SA. The biology of cancer exosomes: insights and new perspectives. Cancer Res. 2017;77:6480-8.

14. Hoshino A, Costa-Silva B, Shen TL, Rodrigues G, Hashimoto A, Tesic Mark $M$, et al. Tumour exosome integrins determine organotropic metastasis. Nature. 2015:527:329-35.

15. Mohrmann L, Huang HJ, Hong DS, Tsimberidou AM, Fu S, Piha-Paul SA, et al. Liquid biopsies using plasma exosomal nucleic acids and plasma cell-free DNA compared with clinical outcomes of patients with advanced cancers. Clin Cancer Res. 2018:24:181-8.

16. Krug AK, Enderle D, Karlovich C, Priewasser T, Bentink S, Spiel A, et al. Improved EGFR mutation detection using combined exosomal RNA and circulating tumor DNA in NSCLC patient plasma. Ann Oncol. 2018;29:700-6.

17. Hur JY, Kim HJ, Lee JS, Choi CM, Lee JC, Jung MK, et al. Extracellular vesicle-derived DNA for performing EGFR genotyping of NSCLC patients. Mol Cancer. 2018:17:15

18. Castellanos-Rizaldos E, Grimm DG, Tadigotla V, Hurley J, Healy J, Neal PL, et al. Exosome-based detection of EGFR T790M in plasma from non-small cell lung cancer patients. Clin Cancer Res. 2018;24:2944-50.

19. Thakur BK, Zhang H, Becker A, Matei I, Huang Y, Costa-Silva B, et al. Double-stranded DNA in exosomes: a novel biomarker in cancer detection. Cell Res. 2014;24:766-9.

20. Wan $Y$, Liu B, Lei H, Zhang B, Wang Y, Huang $H$, et al. Nanoscale extracellular vesicle-derived dna is superior to circulating cell free DNA for mutation detection in early-stage non-small cell lung cancer. Ann Oncol. 2018. https://doi.org/10.1093/annonc/mdy458.

21. Kahlert C, Melo SA, Protopopov A, Tang J, Seth S, Koch M, et al. Identification of double-stranded genomic DNA spanning all chromosomes with mutated KRAS and p53 DNA in the serum exosomes of patients with pancreatic cancer. J Biol Chem. 2014;289:3869-75.

22. Fernando MR, Jiang C, Krzyzanowski GD, Ryan WL. New evidence that a large proportion of human blood plasma cell-free DNA is localized in exosomes. PLoS ONE. 2017;12:e0183915. 
23. Sheikine Y, Rangachari D, McDonald DC, Huberman MS, Folch ES, VanderLaan PA, et al. EGFR testing in advanced non-small-cell lung cancer, a mini-review. Clin Lung Cancer. 2016. https://doi.org/10.1016/j. cllc.2016.05.016.

24. Sequist LV, Waltman BA, Dias-Santagata D, Digumarthy S, Turke AB, Fidias $P$, et al. Genotypic and histological evolution of lung cancers acquiring resistance to EGFR inhibitors. Sci Transl Med. 2011;3:75ra26.

25. Sanmamed MF, Fernandez-Landazuri S, Rodriguez C, Zarate R, Lozano MD, Zubiri L, et al. Quantitative cell-free circulating BRAFV600E mutation analysis by use of droplet digital PCR in the follow-up of patients with melanoma being treated with BRAF inhibitors. Clin Chem. 2015;61:297-304.

26. Watanabe M, Kawaguchi T, Isa S, Ando M, Tamiya A, Kubo A, et al. Ultrasensitive detection of the pretreatment EGFR T790M mutation in nonsmall cell lung cancer patients with an EGFR-activating mutation using droplet digital PCR. Clin Cancer Res. 2015;21:3552-60.

27. Pierson-Perry J, Vaks J, Durham A, Fischer C, Gutenbrunner C, Hillyard D, et al. Evaluation of detection capability for clinical laboratory measurement procedures; approved guideline, ed 2. CLSI Document EP17-A2. 2012.

28. Jiang P, Chan CW, Chan KC, Cheng SH, Wong J, Wong WW, et al. Lengthening and shortening of plasma DNA in hepatocellular carcinoma patients. Proc Natl Acad Sci USA. 2015;112:E1317-25.

29. Jiang P, LO YMD. The long and short of circulating cell-free DNA and the ins and outs of molecular diagnostics. Trends Genet. 2016;32:360-71.

30. Underhill HR, Kitzman JO, Hellwig S, Welker NC, Daza R, Baker DN, et al. Fragment length of circulating tumor DNA. PLoS Genet. 2016;12:e1006162.

31. Buder A, Hochmair MJ, Schwab S, Bundalo T, Schenk P, Errhalt P, et al. Cell-free plasma DNA-guided treatment with osimertinib in patients with advanced EGFR-mutated NSCLC. J Thorac Oncol. 2018;13:821-30.
32. Buder A, Setinek U, Hochmair MJ, Schwab S, Kirchbacher K, Keck A, et al. EGFR mutations in cell-free plasma DNA from patients with advanced lung adenocarcinoma: improved detection by droplet digital PCR. Target Oncol. 2019. https://doi.org/10.1007/s11523-019-00623-x.

33. Gerlinger M, Rowan AJ, Horswell S, Math M, Larkin J, Endesfelder D, et al. Intratumor heterogeneity and branched evolution revealed by multiregion sequencing. N Engl J Med. 2012;366:883-92.

34. Ge Q, Zhou Y, Lu J, Bai Y, Xie X, Lu Z. miRNA in plasma exosome is stable under different storage conditions. Molecules. 2014;19:1568-75.

35. Barrett AN, Thadani HA, Laureano-Asibal C, Ponnusamy S, Choolani M. Stability of cell-free DNA from maternal plasma isolated following a single centrifugation step. Prenat Diagn. 2014;34:1283-8.

36. Malentacchi F, Pizzamiglio S, Verderio P, Pazzagli M, Orlando C, Ciniselli $\mathrm{CM}$, et al. Influence of storage conditions and extraction methods on the quantity and quality of circulating cell-free DNA (ccfDNA): the SPIDIADNAplas External Quality Assessment experience. Clin Chem Lab Med. 2015;53:1935-42.

37. Huang WL, Chen YL, Yang SC, Ho CL, Wei F, Wong DT, et al. Liquid biopsy genotyping in lung cancer: ready for clinical utility? Oncotarget. 2017;8:18590-608.

38. Ansari J, Yun JW, Kompelli AR, Moufarrej YE, Alexander JS, Herrera GA, et al. The liquid biopsy in lung cancer. Genes Cancer. 2016;7:355-67.

\section{Publisher's Note}

Springer Nature remains neutral with regard to jurisdictional claims in published maps and institutional affiliations.
Ready to submit your research? Choose BMC and benefit from:

- fast, convenient online submission

- thorough peer review by experienced researchers in your field

- rapid publication on acceptance

- support for research data, including large and complex data types

- gold Open Access which fosters wider collaboration and increased citations

- maximum visibility for your research: over 100M website views per year

At BMC, research is always in progress.

Learn more biomedcentral.com/submissions 